\title{
Glycosylation of Mouse DPP4 Plays a Role in Inhibiting Middle East Respiratory Syndrome Coronavirus Infection
}

\author{
(D) Kayla M. Peck, ${ }^{\text {a }}$ Adam S. Cockrell, ${ }^{\text {b }}$ Boyd L. Yount, ${ }^{\text {c }}$ Trevor Scobey, ${ }^{\text {c }}$ Ralph S. Baric, ${ }^{\text {c,d }}$ Mark T. Heise ${ }^{\text {b,d }}$ \\ Departments of Biology, ${ }^{a}$ Genetics, ${ }^{b}$ Epidemiology, ${ }^{c}$ and Microbiology and Immunology, ${ }^{\text {d }}$ University of North Carolina-Chapel Hill, Chapel Hill, North Carolina, USA
}

Middle East respiratory syndrome coronavirus (MERS-CoV) utilizes dipeptidyl peptidase 4 (DPP4) as an entry receptor. Mouse DPP4 (mDPP4) does not support MERS-CoV entry; however, changes at positions 288 and 330 can confer permissivity. Position 330 changes the charge and glycosylation state of mDPP4. We show that glycosylation is a major factor impacting DPP4 receptor function. These results provide insight into DPP4 species-specific differences impacting MERS-CoV host range and may inform MERS-CoV mouse model development.

\begin{abstract}
Coror oronaviruses are a diverse family of single-stranded, positivesense RNA viruses that have frequently undergone host range expansion events. While coronaviruses have expanded their host range into humans multiple times over the course of their evolutionary history, two recent events have resulted in the emergence of highly pathogenic epidemic strains. First, severe acute respiratory syndrome coronavirus (SARS-CoV) emerged into the human population in 2003 and infected over 8,000 people before finally being contained by aggressive public health intervention strategies. More recently in 2012, Middle East respiratory syndrome coronavirus (MERS-CoV) emerged from its zoonotic host species into humans, resulting in severe disease and a $38 \%$ mortality rate. MERS-CoV likely originated from a bat reservoir species, as evidenced by the identification of closely related MERS-CoV-like viruses in bats $(1,2)$, although current hypotheses suggest that a camel intermediate host also played an important role in the host range expansion event.

The functional receptor for MERS-CoV was recently identified as dipeptidyl peptidase 4 (DPP4) (3). Interestingly, while MERSCoV can utilize human, bat, and camel DPP4 $(14,15)$, traditional small animal models are nonpermissive, including mice $(4,5)$, ferrets (6), and hamsters $(7,14)$. The relevance of MERS-CoV as an emerging pathogen and the importance of small animal models for studying pathogenesis and for developing vaccines and therapeutics led us to identify the determinants of interactions between the MERS-CoV receptor binding domain (RBD) and mouse DPP4 (mDPP4). Interactions between DPP4 and the MERS-CoV RBD are primarily restricted to blades IV and V of the DPP4 Nterminal $\beta$-propeller domain $(8,9)$. Recently, we found that two key residues in $\mathrm{mDPP} 4$ (A288L and T330R) could permit infection by MERS-CoV when mutated to the human DPP4 (hDPP4) amino acids (4). These residues lie within blades IV and V of the $\beta$-propeller domain $(8,9)$. The importance of A288L can be understood by recognizing that there is a strong hydrophobic region in the MERS-CoV RBD that engages the equivalent hDPP4 residue (L294) (9). In fact, all permissive DPP4 orthologs have a leucine residue at this site (i.e., bat, camel, human, and marmoset). This interaction, however, is altered in $\mathrm{mDPP} 4$, potentially making this hydrophobic region less amenable to interacting with the MERS-CoV RBD.

On blade IV, the T330R substitution in $\mathrm{mDPP} 4$ regulates two potentially critical virus-host cell receptor interaction events. First, arginine 330 provides a highly conserved charge that is pres-
\end{abstract}

ent in all known permissive hosts but missing from all known nonpermissive hosts (Fig. 1A). In hDPP4, the interaction between this residue (R336, relative to hDPP4 numbering) and the MERSCoV RBD Y499 has been previously noted as a key interaction (8, 9). The absence of this interaction could be a primary factor behind the lack of permissivity of mDPP4, as well as other nonpermissive DPP4 orthologs. Second, the T330R mutation knocks out an NXT glycosylation motif in mDPP4. Western blot analysis is consistent with the loss of glycosylation at this site, as evidenced by an $\sim 2.5$-kDa downward shift in the mDPP4 T330R mutant (Fig. 1B). Considering these two potentially important effects, we hypothesized that either the introduction of the conserved charge or the removal of glycosylation was crucial for regulating mDPP4 permissivity to MERS-CoV infection.

To test the impact of glycosylation versus charge on the ability of mDPP4 to support infection by MERS-CoV, we generated a panel of DPP4 mutants (Fig. 1C and D) contained within the 945 RRRE expression vector, a lentiviral vector derived from pTK945. DPP4 constructs were expressed in human embryonic kidney 293T (HEK 293T) cells that lack detectable expression of endogenous hDPP 4 (10). At $18 \mathrm{~h}$ posttransfection with $3 \mu \mathrm{g}$ of the DPP4 expression plasmid, cells were infected with rMERS-CoVRFP which encodes tomato red fluorescent protein in place of ORF5 (11). Cells were imaged $\sim 24 \mathrm{~h}$ postinfection to assess the number of positive cells as a readout for MERS-CoV infection.

A set of hDPP4 mutants were generated and assayed for permissivity to MERS-CoV infection in order to first assess the importance of glycosylation versus charge in the human context. We generated two mutants: one that included a glycosylation site and

Received 2 December 2014 Accepted 28 January 2015

Accepted manuscript posted online 4 February 2015

Citation Peck KM, Cockrell AS, Yount BL, Scobey T, Baric RS, Heise MT. 2015 Glycosylation of mouse DPP4 plays a role in inhibiting Middle East respiratory syndrome coronavirus infection. J Virol 89:4696 - 4699. doi:10.1128/JVI.03445-14. Editor: D. S. Lyles

Address correspondence to Ralph S. Baric, rbaric@unc.edu, or Mark T. Heise, mark_heisem@med.unc.edu.

R.S.B. and M.T.H. contributed equally to this work.

Copyright $\odot$ 2015, American Society for Microbiology. All Rights Reserved. doi:10.1128/JVI.03445-14 


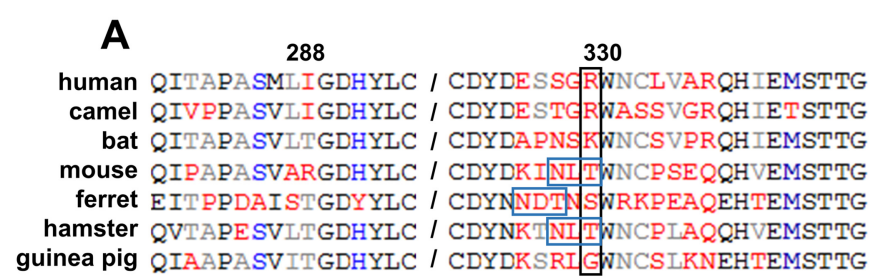

$\square$ Conserved positive charge Glycosylation site

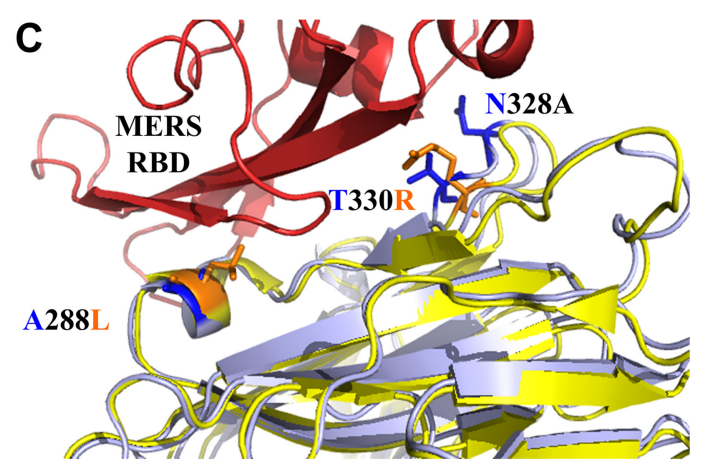

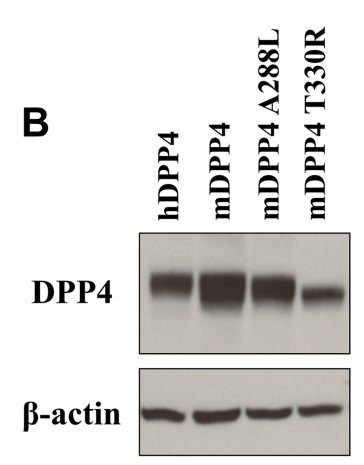

D

\begin{tabular}{|c|c|c|}
\hline Construct & Glycosylated? & $\begin{array}{c}\text { Conserved } \\
\text { positive charge? }\end{array}$ \\
\hline hDPP4 & - & $\mathrm{X}$ \\
\hline $\mathrm{hDPP} 4+$ gly & $\mathrm{X}$ & - \\
\hline $\mathrm{hDPP} 4 \mathrm{R} 336 \mathrm{~T}$ & - & - \\
\hline $\mathrm{mDPP} 4$ & $\mathrm{X}$ & - \\
\hline $\mathrm{mDPP} 4 \mathrm{~A} 288 \mathrm{~L}$ & $\mathrm{X}$ & - \\
\hline $\mathrm{mDPP} 4 \mathrm{~N} 328 \mathrm{~A}$ & - & - \\
\hline $\mathrm{mDPP} 4 \mathrm{~T} 330 \mathrm{R}$ & - & $\mathrm{X}$ \\
\hline $\mathrm{mDPP4}$ A288L, N328A & - & - \\
\hline $\mathrm{mDPP} 4 \mathrm{~A} 288 \mathrm{~L}, \mathrm{~T} 330 \mathrm{R}$ & - & $\mathrm{X}$ \\
\hline
\end{tabular}

FIG 1 Is charge or glycosylation important for mediating mouse DPP4 permissivity? (A) MEGA6 protein sequence alignment of DPP4 for various permissive (human, camel, and bat) and nonpermissive (mouse, ferret, hamster, and guinea pig) species, visualized in GeneDoc. Residue numbers are relative to mDPP4. The mutation T330R in mDPP4 introduces a conserved positive charge for permissive hosts but also knocks out a glycosylation site. NCBI accession numbers are as follows: human, NP_001926.2; camel, AIG55259; bat, AGF80256.1; mouse, NP_034204.1; ferret, ABC72084.1; hamster, AIG55262.1; guinea pig, XP_003478612.2. (B) The downward shift in the mDPP4 T330R band is consistent with the removal of glycosylation. Western blot protocol followed that described by Cockrell et al. (4). (C) Structure of hDPP4 (yellow) complexed with the MERS-RBD (red) (PDB code 4L72) visualized using PyMOL Molecular Graphics System, version 1.6.0.0 (Schrodinger, LLC). mDPP4 (blue), threaded through i-TASSER (13), is overlaid to show the key mutations: A288L, T330R, and N328A. Blue indicates wild-type mDPP4 residues, while orange indicates the human amino acid identity. (D) DPP4 constructs used. Whether they are glycosylated at residue 328 and whether the conserved positive arginine is present at residue 330 (numbered relative to mDPP4) are also shown.

one that removed the charge. First, we swapped the three residues of the NLT mDPP4 putative glycosylation site with residues 334 to 336 of hDPP4 (hDPP4+gly). This addition shows a severe reduction in infection (Fig. 2A and B), with an upward shift in the
Western blot band consistent with successful introduction of the glycosylation site (Fig. 2C). However, this mutation impacts both the glycosylation site and the charged 336 residue (aligning to residue 330 in mDPP4) (Fig. 1A). Therefore, our second mutant
A

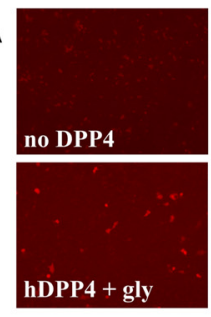

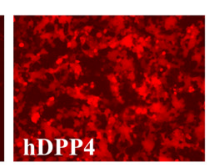

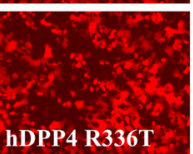

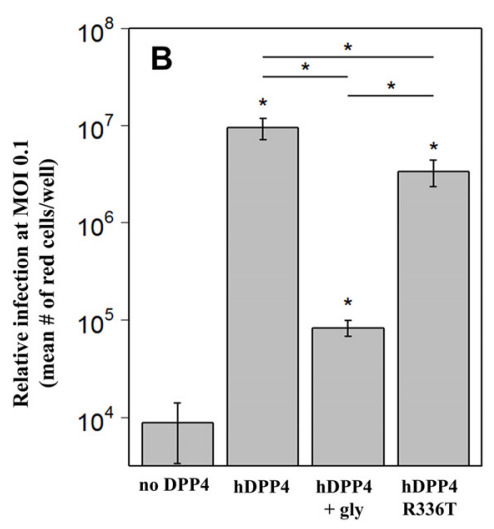

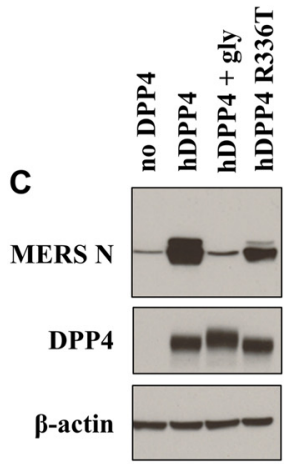

C

FIG 2 Glycosylation can act to dramatically reduce infection by MERS-CoV. (A) HEK 293T cells were transfected with each DPP4 construct and infected with rMERS-CoV-RFP at a multiplicity of infection (MOI) of 1 at $\sim 18 \mathrm{~h}$ posttransfection. At $\sim 24 \mathrm{~h}$ postinfection, cells were imaged. (B) Cells were transfected as described for panel A and infected with rMERS-CoV-RFP at the following MOIs: hDPP4 and hDPP4 R336T, 0.001; no DPP4 and hDPP4+gly, 0.1. At 24 h postinfection, cells were counted based on red fluorescence, and values were normalized to an MOI of 0.1 . Values represent 3 replicates. All mutants have levels that are statistically greater than those obtained with no DPP4, and all other pairwise comparisons are also statistically significant $\left({ }^{*}, P<0.05\right.$; Student's $t$ test). (C) Western blot analysis for MERS nucleocapsid (N) protein, DPP4, and actin as a loading control. The Western blot protocol followed that described by Cockrell et al. (4). 


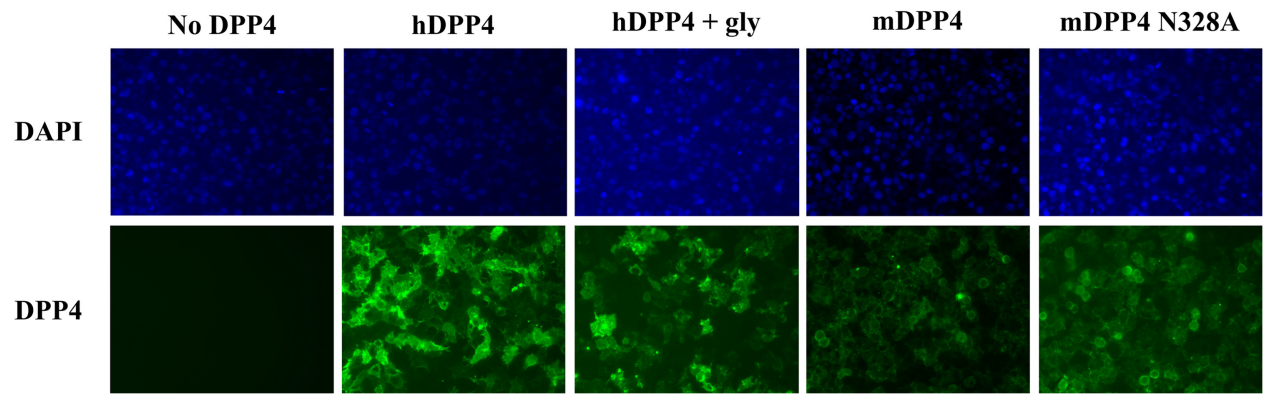

FIG 3 DPP4 and mutant variants are expressed on the surfaces of cells, visible by immunofluorescence. Cells were transfected as described in the legend to Fig. 2A, fixed, and probed with primary goat-anti-DPP4 polyclonal antibody (R\&D Systems) at 1:50 and secondary donkey anti-goat IgG-Alexa Fluor 488 (Life Technologies) at 1:500. Cells were imaged at $40 \times$ for DAPI (30-ms exposure) and DPP4 (160-ms exposure).

contains the R336T mutation by itself, which removes the positive charge without introducing glycosylation. While we did observe a decrease in infection, it was not comparable in magnitude to the decrease seen when glycosylation was included (Fig. 2A and B), suggesting that the presence of a positively charged residue at position 336 is not essential for hDPP4-mediated MERS-CoV infection. Additionally, the presence of glycosylation does not impact the ability of hDPP4+ gly to be expressed on the cell surface (Fig. 3 ). These results show that glycosylation can act to inhibit infection by MERS-CoV and that the positive charge is not a crucial interaction in the context of hDPP4.

In order to directly assess the relative contribution of charge versus glycosylation in the context of mDPP4, we evaluated whether the presence of glycosylation or charge at the 330 site regulates $\mathrm{mDPP} 4$ receptor activity. For these studies, mutations were evaluated singly and in the presence of the secondary mutation (A288L), which is essential for high levels of MERS-CoV re- ceptor activity. Importantly, introduction of the charged residue at 330 simultaneously destroys the glycosylation site, preventing us from testing whether the presence of the charged residue at 330 can enhance mDPP4 receptor activity in the presence of a glycosylation site. However, it is possible to remove the glycosylation site without introducing a charged residue with the mutation N328A, which disrupts the N of the NXT motif (Fig. 1A and D). When we assessed the N328A mutant in the context of the A288L background, we observed high levels of infection (Fig. 4A) that are not statistically different from those obtained with mDPP4 A288L, T330R (Fig. 4B). Both glycosylation knockout mutants have levels that are statistically greater than mDPP4 but statistically less than hDPP4 (Fig. 4B). All mutants containing the T330R or N328A mutation show an $\sim 2.5-\mathrm{kDa}$ downward shift in the Western blot, consistent with the loss of glycosylation (Fig. 4C). Importantly, surface staining for MDPP4 and hDPP4 showed that all derivatives of the DPP4 receptors are expressed at the cell sur-
A
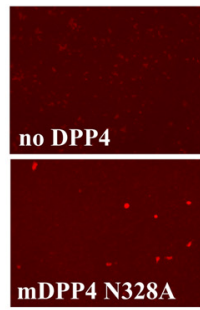

B

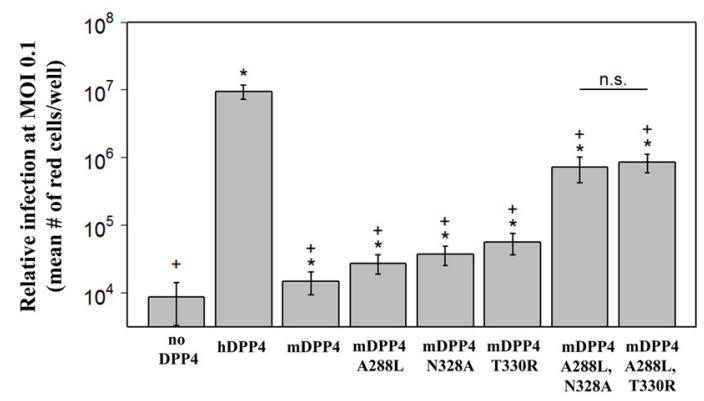

hDPP4
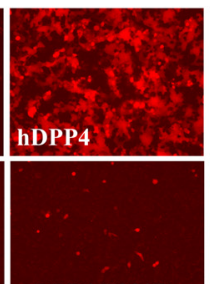

mDPP4 T330R
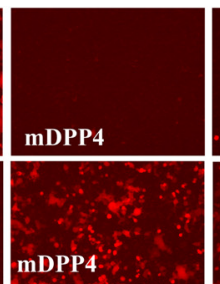

A288L, N328A

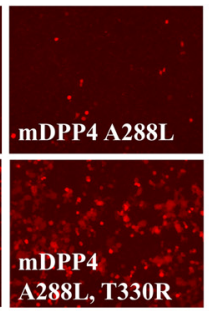

C
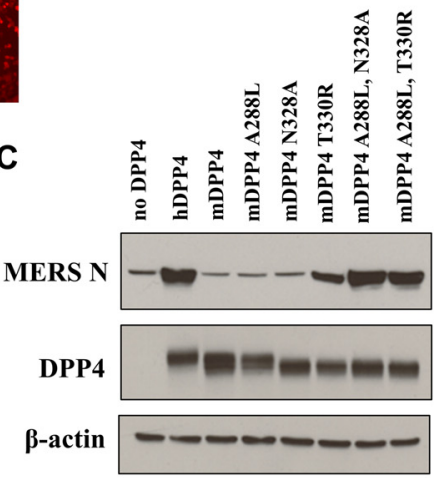

FIG 4 Glycosylation, rather than charge, is a key determinant of mouse DPP4 permissivity to MERS-CoV. (A) Cells were transfected and infected following the protocol detailed in the legend to Fig. 2A. Neither mDPP4 N328A nor mDPP4 T330R can confer permissivity to MERS-CoV; however, both result in strong levels of infection when coupled with A288L. (B) Red cell counts were calculated as for Fig. 2B with the following MOIs: hDPP4, 0.001; mDPP4, mDPP4 A288L, mDPP4 N328A, mDPP4 T330R, and no DPP4, 0.1; mDPP4 A288L, N328A and mDPP4 A288L, T330R, 0.01. Values for all DPP4 constructs are significantly greater than those for no DPP4 and mDPP4 ( ${ }^{*}, P<0.05$; Student's $t$ test) and significantly less than that for hDPP4 (,$+ P<0.05$; Student's $t$ test); however, the values for mDPP4 A288L, N328A and mDPP4 A288L, T330R are not statistically different from each other (n.s. [not significant], $P<0.05$; Student's $t$ test). (C) Western blot analysis for MERS nucleocapsid (N) protein, DPP4, and actin as a loading control. The Western blot protocol followed that described by Cockrell et al. (4). 
face and available to interact with the MERS-CoV RBD (Fig. 3). Together, these results indicate that removal of the glycosylation site, rather than addition of the charged residue at position 330, is responsible for regulating the ability of MERS-CoV to utilize mDPP4 as a functional receptor. The secondary mutation, A288L, also plays an important role in MERS-CoV permissivity due to the fact that high levels of infection are seen only when the glycosylation mutations are combined with the A288L substitution (Fig. 4A and $\mathrm{B}$ ). Together, this suggests that while glycosylation is an important barrier, its removal is not sufficient to permit infection in the absence of the A-to-L modification at position 288 .

The importance of glycosylation in the interactions between coronaviruses and host cell receptors was recognized previously. For example, the introduction of a glycosylation site into human aminopeptidase N (APN) prevents human coronavirus 229E from utilizing it as a receptor (12). For MERS-CoV, it is possible that glycosylation can act as a broader determinant of DPP4-mediated host range, since other nonpermissive hosts (i.e., ferrets and hamsters) also have a nonconserved glycosylation site in the region of DPP4 that interacts with the MERS-RBD (Fig. 1A). In the context of a small animal model, the presence of the glycosylation site at position 330 may sterically hinder multiple interacting residues between the MERS-CoV RBD and mDPP4, complicating the generation of a mouse-adapted strain. Therefore, it may be necessary to partially or fully humanize mDPP4 to achieve in vivo MERS-CoV replication. Additionally, the finding that changes in both blades of $\mathrm{MDPP} 4$ are crucial for mediating permissivity to MERS-CoV (Fig. 4A) has two major implications. First, it may help inform future studies in other nonpermissive hosts, particularly since single amino acid changes have not resulted in successful infections (e.g., ferrets) (data not shown). Second, it suggests that circulating MERS-like coronaviruses cannot expand their host range into mice and possibly other rodent species with just one change. Rather, extensive remodeling of the MERS-CoV RBD is likely required for it to successfully utilize nonpermissive DPP4 orthologs as receptors, especially if glycosylation acts to block infection in these alternate species. Presumably, the modifications that would allow the MERS-CoV RBD to utilize mDPP4 and other orthologs would likely attenuate or even ablate its ability to utilize hDPP4. Overall, by understanding the biochemical determinants that mediate MERS-CoV utilization of DPP4 orthologs, we can begin to characterize the selective pressures leading up to host range expansion events, with the broader goal of being able to predict future emergences.

\section{ACKNOWLEDGMENTS}

We thank members of the Heise and Baric laboratories for review of the manuscript. We thank Jesica Swanstrom for providing the primary mouse polyclonal antiserum.

K.M.P. is supported on an NSF GRFP. This work was supported by grants NIH HHSN272201000019I-HHSN27200003-Task A57 and U19 AI109761.

\section{REFERENCES}

1. Annan A, Baldwin HJ, Corman VM, Klose SM, Owusu M, Nkrumah EE, Badu EK, Anti P, Agbenyega O, Meyer B, Oppong S, Sarkodie YA,
Kalko EK, Lina PH, Godlevska EV, Reusken C, Seebens A, GlozaRausch F, Vallo P, Tschapka M, Drosten C, Drexler JF. 2013. Human betacoronavirus 2c EMC/2012-related viruses in bats, Ghana and Europe. Emerg Infect Dis 19:456-459. http://dx.doi.org/10.3201/eid1903.121503.

2. Memish ZA, Mishra N, Olival KJ, Fagbo SF, Kapoor V, Epstein JH, Alhakeem R, Durosinloun A, Al Asman M, Islam A, Kapoor A, Briese AT, Daszak P, Al Rabeeah AA, Lipkin WI. 2013. Middle East respiratory syndrome coronavirus in bats, Saudi Arabia. Emerg Infect Dis 19:18191923. http://dx.doi.org/10.3201/eid1911.131172.

3. Raj VS, Mou H, Smits SL, Dekkers DH, Müller MA, Dijkman R, Muth D, Demmers JA, Zaki A, Fouchier RA, Thiel V, Drosten C, Rottier PJ, Osterhaus AD, Bosch BJ, Haagmans BL. 2013. Dipeptidyl peptidase 4 is a functional receptor for the emerging human coronavirus-EMC. Nature 495:251-254. http://dx.doi.org/10.1038/nature12005.

4. Cockrell AS, Peck KM, Yount BL, Agnihothram SS, Scobey T, Curnes NR, Baric RS, Heise MT. 2014. Mouse dipeptidyl peptidase 4 is not a functional receptor for Middle East respiratory syndrome coronavirus infection. J Virol 88:5195-5199. http://dx.doi.org/10.1128/JVI.03764-13.

5. Coleman CM, Matthews KL, Goicochea L, Frieman MB. 2014. Wildtype and innate immune-deficient mice are not susceptible to the Middle East respiratory syndrome coronavirus. J Gen Virol 95:408-412. http://dx .doi.org/10.1099/vir.0.060640-0.

6. Raj SV, Smits SL, Provacia LB, van den Brand JM, Wiersma L, Ouwendijk WJ, Bestebroer TM, Spronken MI, van Amerongen G, Rottier PJ, Fouchier RA, Bosch BJ, Osterhaus AD, Haagmans BL. 2014. Adenosine deaminase acts as a natural antagonist for dipeptidyl peptidase 4-mediated entry of the Middle East respiratory syndrome coronavirus. J Virol 88:1834-1838. http://dx.doi.org/10.1128/JVI.02935-13.

7. de Wit E, Prescott J, Baseler L, Bushmaker T, Thomas T, Lackemeyer MG, Martellaro C, Milne-Price S, Haddock E, Haagmans BL, Fedmann H, Munster VJ. 2013. The Middle East respiratory syndrome coronavirus (MERS-CoV) does not replicate in Syrian hamsters. PLoS One 8:e69127. http://dx.doi.org/10.1371/journal.pone.0069127.

8. Lu G, Hu Y, Want Q, Qi J, Gao F, Li Y, Zhang Y, Zhang W, Yuan Y, Bao J, Zhang B, Shi Y, Yan J, Gao GF. 2013. Molecular basis of binding between novel human coronavirus MERS-CoV and its receptor CD26. Nature 500:227-231. http://dx.doi.org/10.1038/nature12328.

9. Wang N, Shi X, Jiang L, Zhang S, Wang D, Tong P, Guo D, Fu L, Cui Y, Liu X, Arledge KC, Chen YH, Zhang L, Wang X. 2013. Structure of MERS-CoV spike receptor-binding domain complexed with human receptor DPP4. Cell Res 23:986-993. http://dx.doi.org/10.1038/cr.2013.92.

10. Zhao G, Du L, Ma C, Li Y, Li L, Poon VK, Wang L, Yu F, Zheng BJ, Jiang S, Zhou Y. 2013. A safe and convenient pseudovirus-based inhibition assay to detect neutralizing antibodies and screen for viral entry inhibitors against the novel human coronavirus MERS-CoV. Virology J 10: 266. http://dx.doi.org/10.1186/1743-422X-10-266.

11. Scobey T, Yount BL, Sims AC, Donaldson EF, Agnihothram SS, Menachery VD, Graham RL, Swanstrom J, Bove PF, Kim JD, Grego S, Randell SH, Baric RS. 2013. Reverse genetics with a full-length infectious cDNA of the Middle East respiratory syndrome coronavirus. Proc Natl Acad Sci U S A 110:16157-16162. http://dx.doi.org/10.1073 /pnas.1311542110.

12. Wentworth DE, Holmes KV. 2001. Molecular determinants of species specificity in the coronavirus receptor aminopeptidase N (CD13): influence of N-linked glycosylation. J Virol 75:9741-9752. http://dx.doi.org/10 $.1128 /$ JVI.75.20.9741-9752.2001.

13. Zhang Y. 2008. I-TASSER server for protein 3D structure prediction. BMC Bioinformatics 9:40. http://dx.doi.org/10.1186/1471-2105-9-40.

14. van Doremalen N, Miazgowicz KL, Milne-Price S, Bushmaker T, Robertson S, Scott D, Kinne J, McLellan JS, Zhu J, Munster VJ. 2014. Host species restriction of Middle East respiratory syndrome coronavirus through its receptor, dipeptidyl peptidase 4. J Virol 88:9220-9232. http: //dx.doi.org/10.1128/JVI.00676-14.

15. Barlan A, Zhao J, Sarkar MK, Li K, McCray PB, Jr, Perlman S, Gallagher T. 2014. Receptor variation and susceptibility to Middle East respiratory syndrome coronavirus infection. J Virol 88:4953-4961. http: //dx.doi.org/10.1128/JVI.00161-14. 